\title{
Document Legally Authenticated
}

National Cancer Institute

\section{Source}

National Cancer Institute. Document Legally Authenticated. NCI Thesaurus. Code

C142514.

A completion status in which a document has been signed manually or electronically by the individual who is leg ally responsible for that document. (HL7) 